\title{
THE CARTAN-BRAUER-HUA THEOREM FOR MATRIX AND LOCAL MATRIX RINGS
}

\section{ALEX ROSENBERG}

1. Introduction. A well known theorem of Cartan-Brauer-Hua states that any division subring of a division ring $D$ setwise invariant under all inner automorphisms (or more briefly, invariant subring) is either contained in the centre or is $D$ itself. In three recent papers, Hattori [3], Hua [5], and Kasch [7] extended this result to $D_{n}$, an $n \times n$ matrix ring over a division ring $D$, i.e., a simple ring with descending chain condition. Indeed, it was shown that if $n \geqq 2$, any invariant additive subgroup of $D_{n}$ not in the centre contains $\left[D_{n}, D_{n}\right]$, the additive group generated by the commutators $[a, b]=a b-b a$, unless $n=2$ and $D=G F(2)$. From this it follows, of course, that the only invariant subrings are $D_{n}$ or subrings of the centre. It is the purpose of this note to show how some of the computations of the above authors (especially those of Kasch) may be used to study invariant additive subgroups of an $n \times n$ matrix ring $A_{n}(n \geqq 2)$ over an arbitrary associative ring $A$ with unit. Such a ring contains a set of matrix units $e_{i j}$ with the usual multiplication table, $\sum e_{i i}$ is the unit of $A_{n}$, and $A$ is the centralizer of the set of matrix units.

It is well known that the only two-sided ideals of $A_{n}$ are of the form $I_{n}, I$ a two-sided ideal of $A$, and so at least the subgroups $I_{n}$ are invariant. We shall see that the most general type of invariant subgroup $S$, not in the centre, is not too far removed from $I_{n}$; indeed, the off-diagonal entries of the elements of $S$ form a nonzero twosided ideal $I$ and $S$ contains $\left[A_{n}, I_{n}\right]$. In particular, if $A$ is simple the only invariant subrings are in the centre or $A_{n}$ itself. In case $n \geqq 3$ we use our result to rederive the theorems of Jacobson and Rickart [6] on the Lie and Jordan ideals of matrix rings; as well as showing, that if $A$ is simple, the only Lie ideals of $\left[A_{n}, A_{n}\right]$ are in the centre. We also remark that if $A$ has no unit the ideals, and consequently, the invariant (under quasi-inner automorphisms) subgroups may be more complicated: thus if $A^{2} \neq A$,

$$
\left(\begin{array}{ll}
A & A \\
A^{2} & A
\end{array}\right)
$$

Presented to the Society, December 29, 1954; received by the editors February 9 , 1955. 
is a proper two-sided ideal in $A_{2}$ not of the form $I_{2}$. In the last part of this note we apply some of our results to show that if a ring is locally a matrix ring of degree $>2$ over a simple ring with unit, the only invariant subrings are subrings of the centre or the ring itself.

A preliminary study of invariant subrings of general matrix rings has already been carried out in [2], and our results include those obtained there.

2. Matrix rings. We shall first deal with the case $n \geqq 3$. It turns out that in this case it is sufficient to assume only invariance under conjugation by elements $1+\lambda e_{i j}, i \neq j$. Following Dieudonné [1] we call these elements the transvections of $A_{n}$. Then we have

THEOREM 1. Let $A$ be a ring with unit and let $A_{n}$ be the ring of $n \times n$ matrices over it. If $n \geqq 3$, any additive subgroup $S$ of $A_{n}$, not in the centre, ${ }^{1}$ is setwise invariant under all inner automorphisms by the transvections if and only if $S=\left[A_{n}, I_{n}\right]+D$. Here $I$ is a nonzero two-sided ideal of $A$ and $D$ is an additive group of diagonal matrices $\sum \delta_{i} e_{i i}$, with $\delta_{i} \equiv \delta_{j} \bmod I$ and $\delta_{i}+I$ in the centre of $A-I$.

Proof. Let $I$ be the set of all elements of $A$ consisting of the offdiagonal entries of elements of $S$. We begin by showing $I \neq 0$. Suppose $s \in S$ is not in the centre but is a diagonal matrix, $s=\sum \alpha_{h} e_{h h}$. Then for $i \neq j\left(1+\lambda e_{i j}\right) s\left(1-\lambda e_{i j}\right)-s=\left(\lambda \alpha_{j}-\alpha_{i} \lambda\right) e_{i j} \in S$ for every $\lambda$ in $A$. Thus if $\alpha_{i} \neq \alpha_{j}, \lambda=1$ will give a nonzero element of $I$. On the other hand if $s$ is a scalar matrix with $\alpha_{i}=\alpha, \alpha$ is not in the centre of $A$ and so for some $\lambda,[\alpha, \lambda] \neq 0$ which again gives a nonzero element of $I$.

Next we show that if $\alpha \in I$, so is $\lambda \alpha \mu$ for any $\lambda, \mu$ in $A$. There is some $s$ in $S$ for which $\alpha$ is an off-diagonal entry, say $s=\sum \alpha_{i j} e_{i j}$ with $\alpha=\alpha_{h k}, k \neq h$. Since $n \geqq 3$ there is an integer $m \leqq n$ such that $m, k, h$ are all distinct. Then for any two elements $\lambda, \mu \in A$ we have just as in $[7, \mathrm{pp} .184-185]$

$$
\begin{aligned}
& t=\left(1+\lambda e_{h k}\right) s\left(1-\lambda e_{h k}\right)-s=e_{h k} \lambda s-s \lambda e_{h k}-e_{h k} \lambda s \lambda e_{h k} \in S, \\
& u=\left(1+\mu e_{h m}\right) t\left(1-\mu e_{h m}\right)-t=-e_{h m} \mu s \lambda e_{h k}-e_{h k} \lambda s \mu e_{h m} \in S
\end{aligned}
$$

and finally

$$
\left(1+e_{m k}\right) u\left(1-e_{m k}\right)-u=e_{h k} \lambda s \mu e_{h k}=(\lambda \alpha \mu) e_{h k} \in S .
$$

Computing $\left(1+e_{i j}\right) \alpha e_{h k}\left(1-e_{i j}\right)-\alpha e_{h k}$ first for $j \neq h, i=k$ and then for $j=h, i \neq k$, we see that $\alpha e_{h j}, j=1, \cdots, h-1, h+1, \cdots, n$; $\alpha e_{i k}, i=1, \cdots, k-1, k+1, \cdots, n$; are in $S$. Then, applying the same operations to $\alpha e_{h j}$ and $\alpha e_{i k}$ as were applied to $\alpha e_{h k}$ yields $\alpha e_{i j} \in S$

1 The set of scalar matrices with entries in the centre of $A$. 
for every pair $i, j, i \neq j$. Hence $I$ is an additive group and this together with the previous paragraph shows that $I$ is a two-sided ideal in $A$ such that $I e_{i j} \subset S, i \neq j$. Finally for each $\lambda$ in $A$ and $\iota$ in $I$ we have, cf. $[7$, p. 185]

$$
\left(1+\lambda e_{i j}\right) \iota e_{j i}\left(1-\lambda e_{i j}\right)-\iota e_{i j}+\lambda \iota \lambda e_{i j}=\lambda \iota e_{i i}-\iota \lambda e_{j j} \in S .
$$

We thus see that $S$ contains the additive subgroup $T$ generated by $I e_{i j}$ and $\lambda \iota e_{i i}-\iota \lambda e_{j j}$. Furthermore, since $\iota e_{i j}=\left[\iota e_{i j}, e_{j j}\right]$ and $\left[\lambda e_{i j}, \iota e_{j i}\right]$ $=\lambda \iota e_{i i}-\imath \lambda e_{j j}, T \subset\left[A_{n}, I_{n}\right]$. But calculating $\left[\lambda e_{i j}, \iota e_{h k}\right]$ for any $\lambda \in A$, $\iota \in I$ and any integers $i, j, h, k$ shows that in any of the three cases $j=h, i \neq k ; j \neq h, i=k ; j=h, i=k ;\left[\lambda e_{i j}, \iota e_{h k}\right] \in T$, so that $T=\left[A_{n}, I_{n}\right]$. (This last equality was also noted in [8].)

From the definition of $I$ and since $I e_{i j} \subset\left[A_{n}, I_{n}\right]$ it is then clear that if $s \in S, s=t+d$, with $t \in\left[A_{n}, I_{n}\right]$ and $d=\sum \delta_{i} e_{i i}$, a diagonal matrix. Applying the same transformations to $d$ as were applied in the beginning of the proof to show $I \neq 0$, immediately shows that for every $\lambda$ in $A, \lambda \delta_{i}-\delta_{j} \lambda \in I$. Thus $\delta_{i} \equiv \delta_{j} \bmod I$ and $\delta_{i}+I$ is in the centre of $A-I$.

These last calculations also serve to show that conversely, any additive subgroup $\left[A_{n}, I_{n}\right]+D$, with $D$ as in Theorem 1 , is setwise invariant under conjugation by the transvections.

We now turn to the case $n=2$ : It is known from [3], [5], and [7] that some restrictions must be imposed on $A$ if the same results as for $n>2$ are to hold. Thus, for example, if $A=B \oplus C$ with $B=G F(2)$ and if $S$ is any invariant subring of $C_{2}$,

$$
S \oplus\left\{\left(\begin{array}{ll}
0 & 0 \\
0 & 0
\end{array}\right), \quad\left(\begin{array}{ll}
e & 0 \\
0 & e
\end{array}\right),\left(\begin{array}{ll}
e & e \\
e & 0
\end{array}\right),\left(\begin{array}{ll}
0 & e \\
e & e
\end{array}\right)\right\}, e \text { the unit of } B,
$$

would also be invariant. To avoid this situation we assume that $A$ admits the operator $1 / 2$, i.e. for each $\lambda$ in $A$, there is a $\mu$ in $A$ with $2 \mu=\lambda$. Then if we retain the same notation as for $n \geqq 3$ we still have $I \neq 0$ but we are not able to show without further hypotheses that $I$ is a two-sided ideal. Indeed, if $\alpha \in I$, there is an $s \in S$, with $\alpha=\alpha_{21}$ say. Then

$$
\begin{aligned}
& \left(1+\lambda e_{12}\right) s\left(1-\lambda e_{12}\right)-s=t \in S, \\
& \left(1+\mu e_{12}\right) t\left(1-\mu e_{12}\right)-t=-(\lambda \alpha \mu+\mu \alpha \lambda) e_{12} \in S .
\end{aligned}
$$

If we now assume that $S$ is invariant under all inner automorphisms of $A_{2}, \quad\left(e_{12}+e_{21}\right)(\lambda \alpha \mu+\mu \alpha \lambda) e_{12}\left(e_{12}+e_{21}\right)=(\lambda \alpha \mu+\mu \alpha \lambda) e_{21} \in S$. Thus in particular $2 \alpha e_{12}, 2 \alpha e_{21}$ are in $S$. We remark that this together with Theorem 1 gives Theorem 2 of [2]. Furthermore, since $\left(1-e_{11} / 2\right)$ - $2 \alpha e_{12}\left(1+e_{11}\right)=\alpha e_{12} \in S, I$ is an additive subgroup of $A$ admitting the operator $1 / 2$ and closed under $\lambda \iota+\iota \lambda, \lambda \in A, \iota \in I$, i.e., $I$ is a Jordan 
ideal [6, p. 491]. Hence any hypothesis on $A$ that allows us to conclude that the only Jordan ideals of $A$ are two-sided ideals will yield the same results as for $n \geqq 3$. For example, it is sufficient to assume (1) $A$ is commutative; or (2) $A$ is simple [4, Theorem 9]; or (3) $A$ is locally matrix [6, Theorem 11]. We shall, however, impose yet a fourth hypothesis on $A$, namely, we assume that $A$ is generated by its quasi-regular elements. For if $\lambda$ is quasi-regular with quasi-inverse $\lambda^{\prime},\left(1+\lambda e_{11}\right) \alpha e_{12}\left(1+\lambda^{\prime} e_{11}\right)-\alpha e_{12}=\lambda \alpha e_{12} \in S$, cf. [7, p. 185]. Hence $I$ is a two-sided ideal, $I e_{12}$ and $I e_{21}$ are in $S$ and so the same results as for $n>2$ hold. Summing up, we have proved

ThEOREM 1'. Let $A$ be a ring with unit admitting the operator $1 / 2$ and generated by its quasi-regular elements. Then any additive subgroup of $A_{2}$, not in the centre, setwise invariant under all inner automorphisms of $A_{2}$ is of the form $\left[A_{2}, I_{2}\right]+D$, where $I$ is a nonzero two-sided ideal of $A$ and $D$ is an additive group of diagonal matrices $\sum \delta_{i} e_{i i}, \delta_{i} \equiv \delta_{j} \bmod I$, $\delta_{i}+I$ in the centre of $A-I$.

As far as invariant subrings rather than invariant subgroups go, we only remark that every invariant subring not in the centre contains the invariant subring $B(I)$ generated by $\left[A_{n}, I_{n}\right]$. This ring may very well not be an ideal in $A_{n}$ : for example, if $I$ is an ideal in the centre of $A$ with $I^{2}=0,\left[A_{n}, I_{n}\right]=B(I)$ is simply the invariant subring of all matrices in $I_{n}$ with trace 0 . In general $B(I) \supset\left(I^{2}\right)_{n}$ since $\iota e_{i j} \iota^{\prime} e_{j i} \in B(I)$, and indeed $B(I)-\left(I^{2}\right)_{n}$ is a zero ring. However, if $A$ is simple we can show

COROLlaRY 1. If $A$ is a simple ring with unit the only subrings of $A_{n}, n \geqq 2$, invariant under all inner automorphisms of $A_{n}$, are subrings of the centre or $A_{n}$ itself (if $n=2, A$ has characteristic $\neq 2$ ).

Proof. In this case, if $S$ is not in the centre, $S \supset\left[A_{n}, A_{n}\right]$, the case $n=2$ being taken care of by $\left[4\right.$, Theorem 9], and since $A^{2}=A$, $B(A)=A_{n}$.

We remark that Corollary 1 , of course, covers the case of simple rings with descending chain condition, which are not division rings. However, it is also true that several of the examples of simple rings with no minimal one-sided ideals are matrix rings of arbitrary degree over a simple ring with unit. Thus, the simple homomorph of the ring of all linear transformations on an infinite dimensional vector space, the ring of all bounded operators on a separable Hilbert space modulo the completely continuous ones, and factors of type $\mathrm{II}_{1}$ and III are all simple matrix rings. Furthermore, it is possible on the basis of Corollary 1 to prove various theorems about the generation of 
simple matrix rings by $r$ th powers, multiplicative commutators, etc. We shall not do so here, but refer instead to the corresponding results for simple rings with descending chain condition in [3], [5], and [7].

We now show how if $n \geqq 3$, the structure of the Lie and Jordan ideals of $A_{n}$ can be deduced from Theorem 1, thus reproving Theorems 11 and 19 of [6]. We recall that a Lie ideal $\mathfrak{R}$ of $A_{n}$ is an additive subgroup of $A_{n}$ closed under $[a, x]=a x-x a, a \in A_{n}, x \in \mathbb{R}$. A Jordan ideal $\Im$ of $A_{n}$ is an additive subgroup of $A_{n}$ such that for each $a$ in $A$ and $j$ in $\Im, a j+j a=\{a, j\}, j^{2}, j a j$, and $a j a$ are in $\Im$. $\Im$ then also contains $a j b+b j a$ for any $a, b$ in $A[6$, p. 491]. Then we have

CoRollary 2. If $\mathbb{R}$ is a Lie ideal of $A_{n}, n \geqq 3$, not in the centre, there is a nonzero two-sided ideal $I$ in $A$ such that $\left[A_{n}, I_{n}\right] \subset \mathbb{R},[6$, Theorem 19].

Proof. We show that $\mathfrak{R}$ is invariant under conjugation by the transvections. Since $\left(1+\lambda e_{i j}\right) x\left(1-\lambda e_{i j}\right)=x+\left[\lambda e_{i j}, x\right]-\lambda e_{i j} x \lambda e_{i j}$ it will be sufficient to show that for every $a$ in $\mathbb{R}, \lambda$ in $A, \lambda e_{i j} a \lambda e_{i j}, i \neq j$ is in $\mathbb{R}$. Since $n \geqq 3$ there is an integer $k \leqq n$ such that $i, j, k$ are distinct. Then if $a \in \mathcal{R}, a_{1}=\left[e_{i i}-e_{j j}, a\right], a_{2}=\left[e_{j k}, a_{1}\right], a_{3}=\left[\lambda e_{j i}, a_{2}\right], a_{4}=\left[\lambda e_{k i}, a_{3}\right]$ all are in $\mathfrak{l}$ and so $a_{4}=\lambda e_{j i} a \lambda e_{j i} \in \mathfrak{R}$. Hence if $\mathfrak{R}$ is not in the centre of $A_{n}$, Theorem 1 yields the desired result. We note that if $n=2$ and $A$ admits the operator $1 / 2$ it is again possible to show that $\mathbb{R}$ is invariant under conjugation by transvections.

Corollary 3. Let $A$ be a simple ring with unit and let $Z$ be the centre of $A_{n}, n \geqq 3$. Then the Lie ring $\left[A_{n}, A_{n}\right]-\left[A_{n}, A_{n}\right] \cap Z$ is a simple Lie ring.

Proof. Since $\lambda e_{i j}=\left[\lambda e_{i j}, e_{j j}\right]$ and $e_{i i}-e_{j j}=\left[e_{i j}, e_{j i}\right]$ are in $\left[A_{n}, A_{n}\right]$ the same calculations as in the proof of Corollary 2 show that a Lie ideal of $\left[A_{n}, A_{n}\right]$ is invariant under conjugation by transvections, hence the result follows from Theorem 1.

Corollary 3 is a special case of a theorem recently announced by Herstein [4, Theorem 4] and also generalizes Theorem 11 of [5].

CoROllaRy 4. If $n \geqq 3$ any Jordan ideal $\Im$ of $A_{n}$ is an ideal of $A_{n}$, i.e., $\Im=I_{n}[6$, Theorem 11].

Proof. If $a \in \Im$, then $\lambda e_{i j} a \lambda e_{i j} \in \Im$. Furthermore

$$
\lambda e_{i j} a=\left\{e_{i j},\left\{\lambda e_{j j}, a\right\}\right\}-e_{i j} a\left(\lambda e_{j j}\right)-\left(\lambda e_{j j}\right) a e_{i j} \in \Im
$$

and so $\Im$ is invariant under conjugation by transvections. Now if $z \in \Im$ is a centre element, $z=\alpha 1, e_{i i} z e_{i i}=\alpha e_{i i} \in \Im$, so that $\Im$ is not in the centre. Thus the proof of Theorem 1 shows that the entries in 
the $i, j$ th place $(i \neq j)$ of the elements of $\Im$ run through a fixed twosided ideal $I$ in $A$. Now if $a=\sum \alpha_{h k} e_{h k} \in \Im, e_{i i} a e_{i i}=\alpha_{i i} e_{i i} \in \Im$ and so $\left\{\alpha_{i i} e_{i i}, e_{i j}\right\}=\alpha_{i i} e_{i j} \in \Im$ also. Hence the diagonal entries of elements of $\Im$ run through $I$ too and so $\Im=I_{n}$.

3. Local matrix rings. In the remainder of this note we extend Corollary 1 to a ring $R$ which is locally a simple matrix ring of degree $\geqq 3$ in the following sense: every finite number of elements of $R$ is contained in a subring which is a matrix ring of degree $\geqq 3$ over a simple ring with unit.

We begin by recalling that if an element $a$ of a ring $R$ has a quasiinverse $a^{\prime}$ the mapping ${ }^{2} r \rightarrow(1+a) r\left(1+a^{\prime}\right)$ is called a quasi-inner automorphism of $R$. Then if $R$ contains a subring $T$ with unit $e$ and if $a, b \in T$ are inverses of each other, i.e., $a b=b a=e$, the inner automorphism $t \rightarrow a t b$ of $T$ can be extended to the quasi-inner automorphism $r \rightarrow(1-e-a) r(1-e-b)$ of $R$. We then have

THEOREM 2. Let $R$ be a ring which is locally a simple matrix ring of degree $\geqq 3$, then any subring of $R$ setwise invariant under all quasiinner automorphisms of $R$ is either in the centre or all of $R .^{3}$

Proof. Let $S$ be an invariant subring not in the centre of $R$. Thus there is an $s \in S$, and an $r \in R$ such that $[r, s] \neq 0$. Now if $x$ is any element of $R, x, r, s$ can be embedded in a subring $A_{n}, n \geqq 3, A$ simple with unit. Then $S \cap A_{n}$ is a nonzero subring of $A_{n}$, not in the centre of $A_{n}$. Furthermore, since any inner automorphism of $A_{n}$ can be extended to a quasi-inner automorphism of $R, S \cap A_{n}$ is an invariant subring of $A_{n}$ and so by Corollary $1, S \cap A_{n}=A_{n}$. Hence $x \in S, S=R$.

The two most common examples of rings satisfying the hypotheses of Theorem 2 are infinite Kronecker products of matrix algebras and simple rings with minimal one-sided ideals not satisfying the descending chain condition. That the latter are locally matrix of arbitrary degree was proved in Theorem 9 of [6], and since they have only 0 in the centre such rings have no proper invariant subrings.

In addition, we can sharpen Theorem 2 somewhat to include the case of a primitive ring with minimal one-sided ideals $R$. We briefly recall the structure theory of $R$, cf. [6, p. 489] and the references given there: there exists a pair of dual vector spaces $M, N$ over a division ring $D$ relative to an inner product $(x, f), x \in M, f \in N$, such that $R$ may be identified with a ring of linear transformations on $M$ with adjoints on $N$. This ring contains the ring $F=F(M, N)$ of all

${ }^{2}$ Here, 1 is used purely as shorthand; $R$ need not have a unit.

3 The corresponding extensions of the Lie and Jordan results will be found in [6]. 
finite-valued linear transformations on $M$ with adjoints on $N$. Indeed, $F$ is the socle of $R$, i.e. the sum of all the minimal left (or right) ideals, and is known to be a simple ring with minimal ideals. It is also known that $R$ and $F$ satisfy the descending chain condition if and only if $M$ is finite-dimensional, in which case $R=F$ is simple, so that Corollary 1 applies. Our extension of Theorem 2 then may be stated as follows:

COROLLARY 5. Let $R$ be a primitive ring with minimal one-sided ideals not satisfying the descending chain condition. A subring $S$, not in the centre, is setwise invariant under all quasi-inner automorphisms of $R$ by socle elements if and only if $S$ contains the socle.

Proof. If $f$ is a quasi-regular socle element with quasi-inverse $f^{\prime}$, $(1+f) a\left(1+f^{\prime}\right)=a+$ socle element, thus the if part is clear. To prove the converse it clearly suffices to show $S \cap F \neq 0$. Thus let $s \in S$ be an element not in the centre of $R$, then a standard argument, cf. e.g. $[7$, p. 184] shows that there is a vector $x \in M$ such that $x, x s$ are linearly independent. But then there is a $g$ in $N$ such that $(x, g)=0$, $(x s, g)=1$. Now define an element $f$ of $F \subset R$ by $z f=(z, g) x$ for any $z$ in $M$. Then $f^{2}=0$ and so $(1+f) s(1-f)-s=s_{1}$ is in $S \cap F$. Since $z s_{1}=(z, g) x s-\left(z, g+g s^{*}\right) x, s_{1}$ is not zero and the corollary is proved.

Corollary 5 contains Kasch's result that a subring of the ring of all linear transformations on an infinite dimensional vector space not in the centre and invariant under all automorphisms is a dense ring of linear transformations.

Finally, we remark that Corollary 5 also can be proved directly as follows:

Since the dimension of $M>2$ there is a $g_{1} \neq 0$ in $N$ such that $\left(x, g_{1}\right)$ $=\left(x s, g_{1}\right)=0$. Then if $f_{1}$ is given by $z f_{1}=\left(z, g_{1}\right) x s, s_{1} f_{1}=0$. Thus $\left(1+f_{1}\right) s_{1}\left(1-f_{1}\right)-s_{1}=f_{1} s_{1} \in S$ and since $z f_{1} s_{1}=\left(z, g_{1}\right) x s s_{1} \neq 0, f_{1} s_{1}$ is a transformation of the form $z \rightarrow(z, g) x,(x, g)=0$. It can easily be seen that any two such transformations can be carried into each element, and since the socle is generated by these transformations, the corollary is proved.

\section{REFERENCES}

1. J. Dieudonné, Les déterminants sur un corps non commutatif, Bull Soc. Math. France vol. 71 (1943) pp. 27-45.

2. G. Ehrlich, $A$ note on invariant subrings, Proc. Amer. Math Soc. vol. 6 (1955) pp. $470-471$.

3. A. Hattori, On invariant subrings, Jap. J. Math. vol. 21 (1951) pp. 121-130.

4. I. N. Herstein, On the Lie ring of a simple ring, Proc. Nat. Acad. Sci. U.S.A. vol. 40 (1954) pp. 305-306. 
5. L. K. Hua, $A$ note on the total matrix ring over a non-commutative field, Annales de la Société Polonaise de Mathématique vol. 25 (1952) pp. 188-198.

6. N. Jacobson and C. E. Rickart, Jordan homomorphisms of rings, Trans. Amer. Math. Soc. vol. 69 (1950) pp. 479-502.

7. F. Kasch, Invariante Untermoduln des Endomorphismenrings eines Vektorraums, Archiv der Mathematik vol. 4 (1953) pp. 182-190.

8. - Über den Automorphismenring einfacher Algebren, Archiv der Mathematik vol. 6 (1955) pp. 59-65.

NORTHWESTERN UNIVERSITY

\section{CANCELLATION IN DIRECT SUMS OF GROUPS}

\section{ELBERT A. WALKER ${ }^{1}$}

1. Introduction. The purpose of this paper is to consider the following question for groups. If $F \oplus G=F^{\prime} \oplus H$ and $F \cong F^{\prime}$, when is $G \cong H$ ? It is easy to see that for $G$ to be isomorphic to $H$ some additional hypothesis must be given. For example let $C_{i}, i=1,2,3, \ldots$ be cyclic of order two. Let $F=C_{2} \oplus C_{3} \oplus C_{4} \oplus \cdots$, let $G=C_{1}$, let $F^{\prime}=C_{1} \oplus C_{3} \oplus C_{5} \oplus \cdots$, and let $H=C_{2} \oplus C_{4} \oplus C_{6} \oplus \cdots$. Clearly $F \oplus G=F^{\prime} \oplus H$ and $F \cong F^{\prime}$, yet $G$ not $\cong H$. One hypothesis that is suggested by this example is to require that $F$ be finitely generated. In fact, in Kaplansky's book, Infinite Abelian groups, p. 13, the author asks the following question for Abelian groups, called Test Problem III. If $F \oplus G=F^{\prime} \oplus H, F \cong F^{\prime}$, and $F$ is finitely generated, is $G \cong H$ ? The main results of this paper are theorems from which Test Problem III follows as a corollary.

2. Notations. The additive notation for groups will be used. The symbol $F \oplus G$ will denote the direct sum of the two groups $F$ and $G$. The commutator subgroup of a group $G$ will be denoted by $Q(G)$. The symbol $\{S\}$ will denote the group generated by the set $S$ of elements. The order of an element $g$ of $G$ will be written $o(g)$, and $o(S)$ will denote the number of elements in the set $S$. The infinite cyclic group will be represented by $C$, and the cyclic group of order $n$ by $C(n)$. The symbol $Z(G)$ will denote the center of the group $G$.

3. Definitions. Suppose $F \oplus G=F^{\prime} \oplus H$. The set of $F$ components of the elements of $F^{\prime}$ will be denoted by $F_{1}$, and the set of $F$ com-

Presented to the Society, September 2, 1955 under the title The solution of Kaplansky's test problem III; received by the editors October 4, 1955.

1 University Fellow, University of Kansas. 